\title{
Caractérisation Agro-Morphologique De Variétés De Cotonnier (Gossypium Hirsutum) Pour Une Régionalisation Economique Pour La Production Du Coton Au Bénin
}

\author{
Hougni Alexis, PhD \\ Imorou Lucien, Ma \\ Dagoudo Augustin, Ma
}

Centre de Recherches Agricoles Coton et Fibres (CRA-CF), Institut National des Recherches Agricoles du Bénin (INRAB), 01 PB 175 Cotonou,

République du Bénin

\section{Zoumarou-Wallis Nouhoun}

Faculté d'Agronomie (FA), Université de Parakou (UP), BP 123, République du Bénin

\section{Abstract}

To take more account of the terroir effect in varietal choice and address the concerns of cotton farmers who disapprove the agricultural policy of single variety, research on varietal regionalization was initiated during four campaigns, by the Agricultural Research Center for Cotton Fibers. This idea is reinforced by the diversity of agro-ecological zones of the country. The nonrenewal of the variety in extension since the 2002-2003 campaign is also an element of justification concerns of industry players. This study aims to exploit the terroir effect to identify the most suitable varieties for each agroecological zone and meet the needs of industry players including producers and ginners. The experiment was conducted in 2011-2012 on Angaradébou sites, Okpara, Savalou and Ketou respectively representing the zones 1 (Alibori / Atakora); 2 (Borgou / Donga); 3 (Hills) and 4 (Zou / Ouemé Plateau / Mono-Couffo). The adopted experimental device is a Fisher block with four replicate and eight treatments or varieties $\mathrm{H} 279-1$ in extension witness. Other varieties include E 944-2; E 956-2; H 769-5; H 782-3; I 875-3; K 768-3 and $\mathrm{K}$ 787-2.The measured parameters are the earliness of production, seed cotton yield in the field, the weight of 100 seeds and the fiber ginning outturn. Data analysis revealed significant differences between and inside sites for most measured parameters. The results showed that varieties E 956-2 (1983.85 kg / ha), H 769-5 (1820.49 kg / ha), K 768-3 (1735.42 kg/ ha) and H 782-3 
(1533.59 kg / ha) in terms of seed cotton yield in the field and compared their performance to shattering were the best respectively in zones 1, 2, 3 and 4.

Keywords: Benin, agro ecological zone, Cotton variety, regionalization, economic impact.

\section{Resume}

Pour prendre davantage en compte l'effet terroir dans le choix variétal et répondre aux préoccupations des cotonculteurs qui désapprouvent la politique agricole de variété unique, une recherche sur la régionalisation variétale a été initiée durant quatre campagnes, par le Centre de Recherches Agricoles Coton et Fibres. Cette idée est renforcée par la diversité des zones agro écologiques du pays. Le non renouvellement de la variété en vulgarisation depuis la campagne 2002-2003 constitue aussi un élément de justification des préoccupations des acteurs de la filière. Cette étude vise à exploiter l'effet terroir pour identifier les variétés les plus adaptées à chacune des zones agro-écologiques et qui répondent bien aux besoins des acteurs de la filière notamment les producteurs et les égreneurs. L'expérimentation a été menée en 2011-2012 sur les sites de Angaradébou, Okpara, Savalou et Kétou, représentant respectivement les zones 1 (Alibori/Atacora); 2 (Borgou/Donga) ; 3 (Collines) et 4 (Zou/Ouemé-Plateau/Mono-Couffo). Le dispositif expérimental adopté est un Bloc de Fischer avec quatre répétitions

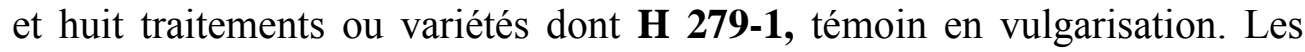
autres variétés sont E 944-2 ; E 956-2 ; H 769-5 ; H 782-3 ; I 875-3 ; K 768 3 et K 787-2. Les paramètres mesurés sont la précocité de production, le rendement coton-graine au champ, le poids de 100 graines, le rendement fibre à l'égrenage. L'analyse des données a révélé des différences significatives inter et intra sites pour la plupart des paramètres mesurés. Les résultats ont montré que les variétés E 956-2 (1983,85 kg/ha), H 769-5 (1820,49 kg/ha), K 768-3 (1735,42 kg/ha) et H 782-3 $(1533,59 \mathrm{~kg} / \mathrm{ha})$ sur le plan du rendement coton-graine au champ et par rapport à leur performance à l'égrenage ont été les meilleures respectivement dans les zones 1, 2, 3 et 4.

Mots clés : Bénin, zone agro écologique, variété de cotonnier, régionalisation, Incidence économique.

\section{Introduction}

Le coton constitue la principale culture de fibres dans le monde. Dans la plupart des pays d'Afrique Zone Franc (AZF) notamment le Bénin, le Burkina Faso, le Mali, le Tchad et le Togo, le coton est à la base du développement et contribue considérablement à l'emploi et aux revenus des populations rurales (Matthess et al., 2005). Dans les pays AZF, les superficies 
cotonnières en 2005 ont été évaluées à 2,6 millions d'hectares, d'après les statistiques officielles du Comité Consultatif International du Coton (CCIC), soit 3\% des superficies cultivées en coton dans le (Esteulle et Perenne, 1996). La production est exportée à plus de 95\% et le groupe des pays d'AZF représentent près de 14\% des exportations mondiales (Hougni, 2009).

La filière coton est l'une des plus importantes de l'agriculture béninoise de par sa contribution à l'entrée de devise et à la création de multiples emplois notamment dans les secteurs du transport, de l'artisanat, de l'industrie et du commerce (Hazard, 2003). Les exportations de coton participent pour 70 à $80 \%$ à la constitution des recettes d'exportation officielle et $35 \%$ des entrées fiscales, hors douane et représente en termes de valeur ajoutée 14\% du PIB (Hougni, 2004). En milieu rural, plusieurs infrastructures sociocommunautaires (centre de santé, écoles, routes, puits, centres de loisirs...etc.) ont été réalisées grâce aux revenues procurés par le coton. Mais, depuis une dizaine d'années la filière connait d'énormes difficultés avec pour conséquence la réduction des emblavures et la diminution de la production. Après une production record de 427000 tonnes en 2004-2005 due aux nombreuses mesures incitatives mises en œuvre par différents programmes de développement de la filière, la production est entrée dans une phase décroissante pour atteindre 160.000 tonnes en 2009-2010 (Kpadé, 2011). Face à cette situation qui est due en partie aux problèmes d'ordres organisationnel et technique notamment la baisse de rendement et des emblavures, le non renouvellement de la variété, les perturbations pluviométriques. La politique «agricole de variété unique » qui perdure depuis plus d'une vingtaine d'années est aussi en partie responsable de cette baisse de production. Le besoin de rechercher des variétés spécifiques et plus adaptées aux conditions des différents terroirs se fait sentir. Cette éventualité de variétés par région est aussi réclamée à tort ou à raison par cotonculteurs. Aussi, Ashokkumar et al., (2011) ont évoqué la possibilité d'amélioration de la rentabilité de la production cotonnière par le choix continu de variétés adaptées aux conditions édaphique et climatique. Ainsi, l'amélioration de la productivité pourrait donc passer par une politique agricole de variétés spécifiques de cotonniers adaptés aux différents écosystèmes du Bénin. Cette étude est une occasion intéressante pour revisiter tout le matériel créé au Bénin et éprouvé durant ces dix dernières années dans le dispositif multi local habituel d'évaluation variétale en milieu contrôlé et en milieu réel. Cette étude entend apporter des éléments de décision au passage de la politique de variété unique à une politique de variétés régionalisées et adaptées à chacune des grandes zones agro écologiques productrices de coton du Bénin. L'objectif général de cette étude est d'améliorer la productivité et la durabilité de la production cotonnière au Bénin à travers la mise à disposition des cotonculteurs de variétés performantes adaptées à chacune de leur zone agro écologique. 


\section{Materiels et methode}

Les expérimentations ont été localisées de façon à couvrir tous les bassins cotonniers du Bénin. Ainsi, quatre (4) parcelles d'expérimentation ont été installées dans les zones de production cotonnière. Les caractéristiques environnementales (MAEP, 2000) des zones agro écologiques se présentent ainsi qu'il suit:

La Zone 1 (Alibori/Atacora), est comprise entre le $10^{\circ} 45^{\prime}$ et $12^{\circ} 00^{\prime}$ latitude Nord et jouit d'un climat de type soudano-sahélien avec une seule saison des pluies et une pluviométrie moyenne annuelle de 700 à 900 $\mathrm{mm}$. Dans cette zone l'essai a été installé à Angaradébou ;

La Zone 2 (Borgou/Donga), est située entre le $8^{\circ} 30^{\prime}$ et $10^{\circ} 45^{\prime}$ latitude Nordet bénéficie d'un climat de type soudano-sahélien à une seule saison de pluie. La pluviométrie est de 600 à $950 \mathrm{~mm}$ répartie sur 80 à 110 jours. Dans cette zone, l'essai a été installé à Okpara ;

La Zone 3 (département des Collines), est comprise entre le $7^{\circ} 45^{\prime}$ et $8^{\circ} 30^{\prime}$ latitude Nord. Elle est caractérisée par un climat de type soudanoguinéen à 2 saisons de pluie, avec une tendance vers le type soudano-sahélien à une seule saison de pluie dans le secteur nord de la zone, la pluviométrie est de 600 à $1400 \mathrm{~mm}$ répartie sur 80 à 110 jours. Cette zone est représentée par le site de Savalou.

La Zone 4 (Zou/Plateau/Couffo) est comprise entre le $6^{\circ} 45^{\prime}$ et $7^{\circ} 45^{\prime}$ latitude Nord, elle jouit d'un climat de type soudano-guinéen à 2 saisons de pluie, la pluviométrie est de 600 à $1400 \mathrm{~mm}$ répartie sur 80 à 110 jours. Cette zone est représentée par le site de recherche de Kétou.

Le matériel végétal est composé de huit (8) variétés éprouvées dans le dispositif des essais multi locaux dont la variété témoin H 279-1, en vulgarisation (tableau 1).

Tableau 1: Matériel végétal et leurs origines

\begin{tabular}{ccc}
\hline Dénomination & Origine génétique & Origine du croisement \\
\hline H 279-1 (Témoin) & Complexe U & Bénin-Togo \\
E 956-2 & A 12 12 Bénin \\
E 944-2 & A 16 16 & Bénin \\
H 769-5 & A 21 & Bénin \\
& G 165 x CR 92- & Bénin \\
H 782-3 & 534 & Bénin \\
I 875-3 & G 165 x CR 92- & Bénin \\
K 768-3 & F 145-2 x F 244-1 & Bénin \\
K 787-2 & G 165 x CR 92- & \\
\hline
\end{tabular}

Source : (CRA-CF, 2011) 
Le Dispositif expérimental est un Bloc de Ficher à 8 Objets (variétés) et 4 répétitions, avec des parcelles élémentaires de 5 lignes de $20 \mathrm{~m}$. Les parcelles ont été installées à Angaradébou, à Okpara, à Savalou et à Kétou. Les semis ont été faits à bonne date et aux écartements de $0,80 \mathrm{~m} \times 0,3 \mathrm{~m}$ à plant.

Les parcelles sont labourées au tracteur. Les labours des sols ont été effectués à Angaradébou, Okpara, Savalou et Kétou respectivement le 27 Mai, le 26 Mai, le 30 Juin et le 09 Juin 2011. Les semis ont lieu à Angaradébou, Okpara, Savalou et Kétou respectivement le 29 Mai, le 11 Juin, le 04 Juillet et le 30 Juin 2011.

La fertilisation est celle recommandée par la recherche, soit la dose de $200 \mathrm{~kg}$

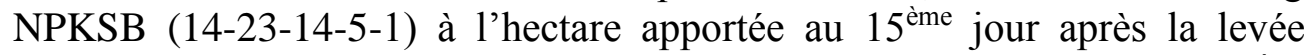
auxquelles il a été associée $50 \mathrm{~kg}$ d'Urée ( $46 \%$ ) à l'hectare appliqué au $40^{\text {ème }}$ jours après la levée.

Les sarclages (02) et sarclo-buttage (01) ont permis de contrôler les mauvaises herbes au niveau de chaque site. Le programme de traitements phytosanitaire est le même dans tous les sites et se présente comme dans le tableau ci-après. Les parcelles sont maintenues relativement propres. La fertilisation est celle recommandée. Des traitements phytosanitaires à intervalle de dix jours, ont permis de préserver les cotonniers des bio-agresseurs. Le programme de traitements phytosanitaire est le même dans tous les sites et se présente comme dans le tableau 2 ci-après.

Tableau 2 : programme de traitement exécuté dans le cadre de l'étude

\begin{tabular}{|c|c|c|c|}
\hline Périodes & Produit commercial & Matières actives & $\begin{array}{l}\text { Doses/ } \\
\text { ha }\end{array}$ \\
\hline T1 : 45 JAL & Tihan 175 O-TEQ & $\begin{array}{l}\text { Flubendiamide } 100 \mathrm{~g} / 1 \text {, spirotétramate } \\
\qquad 75 \mathrm{~g} / 1\end{array}$ & 0,21 \\
\hline $\mathrm{T} 2: 55 \mathrm{JAL}$ & Tihan 175 O-TEQ & $\begin{array}{l}\text { Flubendiamide } 100 \mathrm{~g} / 1 \text {, spirotétramate } \\
\qquad 75 \mathrm{~g} / 1\end{array}$ & 0,21 \\
\hline T3 :65 JAL & Tihan 175 O-TEQ & $\begin{array}{l}\text { Flubendiamide } 100 \mathrm{~g} / 1 \text {, spirotétramate } \\
\qquad 75 \mathrm{~g} / 1\end{array}$ & 0,21 \\
\hline T4 :75 JAL & $\begin{array}{l}\text { Nurelle D36/200 } \\
\text { CE }\end{array}$ & Cyperméthrine-Chlorpyrifos $36-200 \mathrm{~g} / \mathrm{l}$ & $1 / 21$ \\
\hline T5 :85 JAL & Thunder OD 145 & $\begin{array}{l}\text { betacyfluthrine-imidaclopride } 45 \text { - } \\
\qquad 100 \mathrm{~g} / \mathrm{l}\end{array}$ & $1 / 41$ \\
\hline T6 :95 JAL & Thunder OD 145 & $\begin{array}{l}\text { (betacyfluthrine -imidaclopride } 45 \text { - } \\
100 \mathrm{~g} / \mathrm{l})\end{array}$ & $1 / 41$ \\
\hline $\mathrm{T} 7: 105 \mathrm{JAL}$ & Thunder OD 145 & $\begin{array}{l}\text { (betacyfluthrine -imidaclopride 45- } \\
100 \mathrm{~g} / \mathrm{l})\end{array}$ & $1 / 41$ \\
\hline T8 :115 JAL & Thunder OD 145 & $\begin{array}{l}\text { (betacyfluthrine -imidaclopride } 45 \text { - } \\
100 \mathrm{~g} / \mathrm{l} \text { ) }\end{array}$ & $1 / 41$ \\
\hline T9 :125 JAL & Thunder OD 145 & $\begin{array}{l}\text { (betacyfluthrine -imidaclopride } 45 \text { - } \\
100 \mathrm{~g} / \mathrm{l} \text { ) }\end{array}$ & $1 / 41$ \\
\hline
\end{tabular}


Les données collectées concernent essentiellement le rendement en coton-graine, la précocité de production $(\mathrm{P})$, le seed index (poids de 100 graines) et le rendement fibre à l'égrenage. Les récoltes ont été faites sur les lignes centrales des parcelles élémentaires. La première récolte a été faite lorsque les premières variétés ont dépassé les $70 \%$ d'ouverture des capsules et la deuxième à l'ouverture totale des capsules. Ceci a permis d'évaluer la précocité de production (Cf. Formule Ci-dessous). Les rendements en cotongraine ont été obtenus par pesée du coton-graine récolté sur les lignes centrales. Le rendement à l'égrenage, le poids de 100 graines ou seed-index (SI) sont mesurés après égrenage du coton-graine récolté.

$$
P=\frac{R 1}{R T} X 100
$$
Totale.

Avec $P$ : Précocité de production, $R 1:$ première récolte, $R T$ : Récolte

Toutes les données ont été traitées à l'aide d'Excel. L'analyse de variance (ANOVA) des données collectées a été réalisée à l'aide du logiciel Minitab 14. Le test de Scott-Knotta été fait à l'aide de Basic Statistic, pour classer les variétés en des groupes homogènes.

L'étude de la rentabilité économique des nouvelles variétés et donc de la politique de régionalisation a été faite grâce à l'approche par budget partiel. Ce choix s'explique par le fait que le remplacement de la variété en vulgarisation par l'une ou l'autre des nouvelles variétés dans chacune de différentes aires de production n'engendre pas des charges supplémentaires sur toute la chaîne de production. Le modèle économique adopté pour l'étude est celui utilisé par Hougni et al., (1999) dans leurs études sur la proposition d'adoption de la variété H 279-1 actuellement en vulgarisation. Cette étude économique intègre des données agronomiques (rendement coton-graine, rendement fibre à l'égrenage et pourcentage de graine), techniques (pertes à l'usine, prix du kg de coton-graine, de la fibre et de la graine) et du marché international (coût d'une classe de longueur et d'un écart d'indice de jaune).

Plusieurs hypothèses ont aussi été émises, notamment sur la production nationale en coton-graine et sur la part de marché de chacune des aires de production cotonnière. Toutes ces hypothèses sont basées sur les données historiques des vingt (20) dernières campagnes (tableau 3 ).

Tableau 3 : Paramètres technique et financiers pour l'étude économique

\begin{tabular}{c|llllc}
\hline Zones & Pays & Z1 & Z2 & Z3 & Z4 \\
Éléments de calcul & & & & & \\
\hline Hypothèses techniques & 300 & & & & \\
Production nationale (tonne)* & 000 & & & & \\
Part de marché en coton graine (\%)* & 100 & 53 & 26 & 16 & 5 \\
Perte en usine (\%) & 3,0 & 3,0 & 3,0 & 3,0 & 3,0 \\
Hypothèses de prix & & & &
\end{tabular}


Longueur (FCFA/Classe)

$\begin{array}{ccccc}25000 & 250000 & 250000 & 250000 & 250000 \\ 0 & & & & \\ 15000 & 15000 & 15000 & 15000 & 15000 \\ 5000 & 5000 & 5000 & 5000 & 5000 \\ 1130 & 1130 & 1130 & 1130 & 1130 \\ 000 & 000 & 000 & 000 & 000\end{array}$

Indice de jaune (FCFA/Unité)

Fibre (FCFA/Tonne) au $1^{\text {er }}$ fév. 2012

$000 \quad 000$

000

000

$\underline{\text { NB }}$ : la production nationale et la part de marché des zones, sont des moyennes historiques calculées sur les vingt dernières campagnes.

\section{Resultats}

\section{Précocité de production des différentes variétés de cotonnier}

L'analyse de variance a mis en évidence une différence hautement significative entre les sites pour la précocité de production (tableau 4). Dans les zones 1 et 4 , aucune différence significative n'a été observée entre les variétés alors que dans les zones $2(p=0,04)$ et $3(p=0,03)$, des différences significatives ont été notées entre les variétés.

Dans la zone 2, toutes les variétés mises en comparaison sont d'un même niveau de précocité, alors que dans la zone 3, les variétés I 875-3 et H 782-3 ont été les plus précoces. Par contre pour ce qui est des zones 1 et 4 , on ne saurait se prononcer sur la précocité de l'une ou l'autre des variétés mises en comparaison.

Tableau 4: Précocité de production de huit (8) variétés éprouvées de cotonnier dans les différentes zones agro écologiques du Bénin

\begin{tabular}{|c|c|c|c|c|c|c|c|c|}
\hline \multirow{2}{*}{$\begin{array}{l}\text { Site } \\
\text { Varietóc }\end{array}$} & \multicolumn{2}{|c|}{ Zone 1} & \multicolumn{3}{|c|}{ Zone 2} & \multirow{2}{*}{$\begin{array}{r}\text { Zone } 3 \\
\text { écart }\end{array}$} & \multicolumn{2}{|c|}{ Zone 4} \\
\hline & $\mathrm{R} 1 / \mathrm{RT}$ & Écart & $\mathrm{R} 1 / \mathrm{RT}$ & Écart & $\mathrm{R} 1 / \mathrm{RT}$ & & $\mathrm{R} 1 / \mathrm{RT}$ & écart \\
\hline Н 279-1 & 81,90 & - & $54,95 \mathrm{a}$ & - & $88,02 b$ & - & 56,57 & - \\
\hline E 956-2 & 78,36 & $-3,54$ & $50,46 a$ & $-4,49$ & $85,48 b$ & $-2,54$ & 64,47 & 7,90 \\
\hline E 944-2 & 75,72 & $-6,18$ & $50,27 \mathrm{a}$ & $-4,68$ & $87,49 \mathrm{~b}$ & $-0,53$ & 61,54 & 4,97 \\
\hline Н 769-5 & 87,76 & 5,86 & $48,33 a$ & $-6,62$ & $87,54 b$ & $-0,48$ & 56,59 & 0,02 \\
\hline Н 782-3 & 76,58 & $-5,32$ & $48,83 \mathrm{a}$ & $-6,12$ & $89,73 a$ & 1,71 & 62,11 & 5,54 \\
\hline I 875-3 & 89,04 & 7,14 & $48,34 \mathrm{a}$ & $-6,61$ & $90,00 \mathrm{a}$ & 1,98 & 64,80 & 8,23 \\
\hline K 768-3 & 75,35 & $-6,55$ & $48,00 \mathrm{a}$ & $-6,95$ & $85,81 \mathrm{~b}$ & $-2,21$ & 56,42 & $-0,15$ \\
\hline K 787-2 & 75,34 & $-6,56$ & $48,39 a$ & $-6,56$ & $86,65 b$ & $-1,37$ & 61,36 & 4,79 \\
\hline $\mathrm{Nb}$ rép. & \multicolumn{8}{|c|}{4} \\
\hline Ecartyp & \multicolumn{2}{|c|}{12,31} & \multicolumn{3}{|c|}{3,64} & 2,40 & \multicolumn{2}{|r|}{6,38} \\
\hline CV $(\%)$ & & & & 7,32 & & 2,73 & \multirow{2}{*}{\multicolumn{2}{|c|}{10,55}} \\
\hline P.site & \multicolumn{5}{|c|}{0,00} & & & \\
\hline P.variét & \multicolumn{2}{|c|}{0,25} & \multirow{2}{*}{\multicolumn{3}{|c|}{$\begin{array}{c}0,04 \\
*\end{array}$}} & 0,03 & \multicolumn{2}{|r|}{0,16} \\
\hline $\mathrm{F}$ & \multicolumn{2}{|c|}{ Ns } & & & & $*$ & \multicolumn{2}{|r|}{ ns } \\
\hline
\end{tabular}

CV : Coefficient de Variation, ns : non significatif, * : Significatif au seuil de 0,05 , Nbrerep: nombre de répétition, $\mathrm{R} 1$ : première récolte, $\mathrm{RT}$ : récolte totale. Les moyennes suivies des lettres différentes pour les mêmes caractéristiques sont significativement différentes $(\mathrm{p}<0,05)$ selon le test de Scott-Knott et celles suivies de la même lettre ne sont pas significativement différentes $(\mathrm{p}<0,05)$ selon le test. 


\section{Rendement en coton-graine des différentes variétés de cotonnier}

L'analyse de variance du rendement coton-graine au champ a révélé l'existence d'une différence hautement significative $(p=0,00)$ entre les sites (tableau 4). Donc les variétés sont différemment classées d'un site à un autre.

L'analyse de variance a aussi révélé des différences significatives (Zone 2 et4) à hautement significative (Zone 1 et 3 ) entre les variétés (Tableau 5).

Dans la zone 1, on distingue trois groupes homogènes d'après le test Scott-Knott. Le groupe des variétés à rendements les plus élevés: E 956-2, H 782-3 et $\mathrm{H}$ 769-5, le groupe des variétés à moyens rendements : H 279-1, K 768-3, I 875-3 et K 787-2 et le groupe de la variété à faibles rendements : E 944-2.

Dans la zone 2, malgré l'existe de différence significative entre les variétés, un seul groupe homogène se distingue. Les trois variétés de tête sont H 769-5, K 768-3 et K 787-2 pour leurs rendements au champ.

Dans la zone 3, deux groupes homogènes ont été identifiés : le groupe des variétés à rendements les plus élevés : K 768-3, E 944-2 et H 769-5 et le groupe des variétés à faibles rendements: H 279-1, H 782-3, I 875-3, E 956-2 et K 787-2.

Dans la zone 4, les variétés se classent en deux groupes homogènes. Le premier groupe est composé des meilleures variétés que sont $\mathrm{H}$ 782-3, E 956-2, I 875-3 et E 944-2 et le deuxième groupe est composé des variétés de même niveau de rendement que le témoin. Il s'agit de : H279-1, K 768-3, H 769-5 et $\mathrm{K}$ 787-2.

Il a été constaté un gradient de rendement du Nord au Sud. Ainsi, les rendements baissent au fur et à mesure que l'on va de la zone 1 à la zone 4 .

Tableau 5: Rendement en coton-graine de huit (8) variétés éprouvées de cotonnier dans les différentes zones agro écologiques.

\begin{tabular}{|c|c|c|c|c|c|c|c|c|}
\hline \multirow{2}{*}{$\begin{array}{l}\text { Site } \\
\text { Variété }\end{array}$} & \multicolumn{2}{|c|}{ Zone 1} & \multicolumn{2}{|c|}{ Zone 2} & \multicolumn{2}{|l|}{ Zone 3} & \multicolumn{2}{|c|}{ Zone 4} \\
\hline & RDT & \% tém & RDT & \% tém & RDT & \% tém & RDT & $\underline{\frac{\%}{0}}$ \\
\hline H 279- & 1702,08 & 100,0 & 1636,46 & 100,0 & 1597,92 & 1000 & 1260,16 & $\overline{100,0}$ \\
\hline 1 & $\mathrm{~b}$ & 0 & $\mathrm{a}$ & 0 & $\mathrm{~b}$ & 0 & $\mathrm{~b}$ & 0 \\
\hline E 956- & 1983,85 & 116,5 & 1486,59 & & 1518,75 & & 1479,69 & 117,4 \\
\hline 2 & $\mathrm{a}$ & 5 & $\mathrm{a}$ & 90,84 & $\mathrm{~b}$ & 95,05 & $\mathrm{a}$ & 2 \\
\hline E 944- & 1393,23 & & 1602,78 & & 1727,08 & 108,0 & 1313,28 & 104,2 \\
\hline 2 & $\mathrm{c}$ & 81,85 & $\mathrm{a}$ & 97,94 & $\mathrm{a}$ & 8 & $\mathrm{a}$ & 2 \\
\hline Н 769- & 1815,10 & 106,6 & 1820,49 & 111,2 & 1715,63 & 107,3 & 1166,41 & \\
\hline 5 & $\mathrm{a}$ & 4 & $\mathrm{a}$ & 5 & $\mathrm{a}$ & 7 & b & 92,56 \\
\hline H 782- & 1856,77 & 109,0 & 1525,00 & & 1560,42 & & 1533,59 & 121,7 \\
\hline 3 & $\mathrm{a}$ & 9 & $\mathrm{a}$ & 93,19 & b & 97,65 & $\mathrm{a}$ & 0 \\
\hline I $875-3$ & 1609,90 & & 1571,53 & & 1552,08 & & 1363,28 & 108,1 \\
\hline & b & 94,58 & a & 96,03 & $\mathrm{~b}$ & 97,13 & $\mathrm{a}$ & 8 \\
\hline K 768- & 1660,94 & & 1710,76 & 104,5 & 1735,42 & 108,6 & 1178,13 & \\
\hline 3 & b & 97,58 & $\mathrm{a}$ & 4 & $\mathrm{a}$ & 0 & b & 93,49 \\
\hline
\end{tabular}




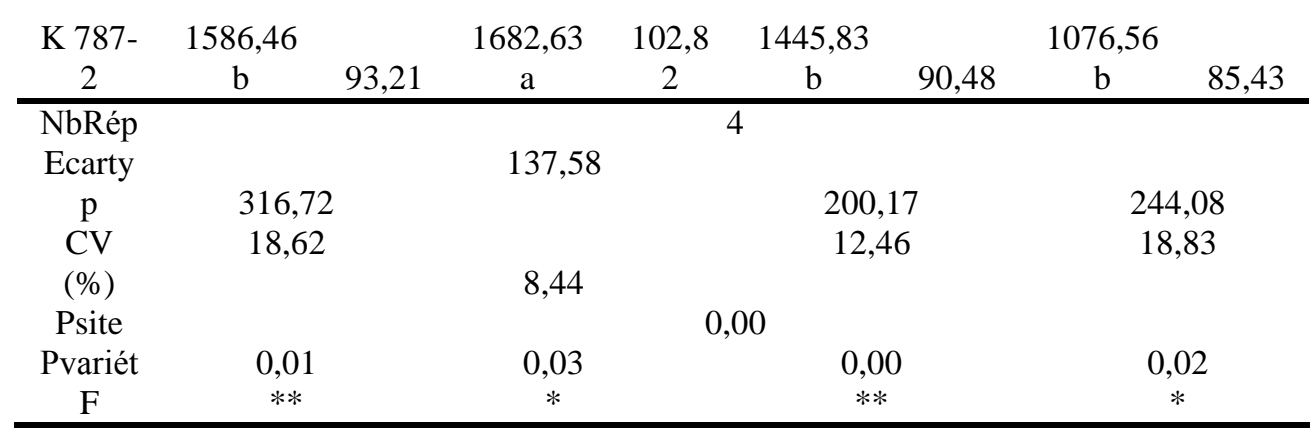

CV : Coefficient de Variation, ns : non significatif, * : Significatif au seuil de $0,05, * *$ :

Hautement significatif au seuil de 0,01 , Nbrerep: nombre de répétition. Les moyennes suivies des lettres alphabétiques différentes $\mathrm{a}$, b et c pour les mêmes caractéristiques sont significativement différentes $(p<0,05)$ d'après le test Scott-Knott et celle suivies de la même lettre pour les mêmes caractéristiques ne sont pas significativement différentes $(\mathrm{p}<0,05) ;$ RDT :rendement, \%tém :par rapport au témoin .

\section{Rendement fibre à l'égrenage (RE) des différentes variétés}

L'analyse de variance des données du rendement à l'égrenage (RE) n'a révélé aucune différence significative entre les sites. Cependant, des différences hautement significatives $(p=0,00)$ ont été observées entre les variétés à l'intérieur d'un même site (tableau 6).Des résultats obtenus, il ressort que :

Dans la zone 1, la variété I 875-3, possède le rendement en fibre le plus élevé. Elle est suivie de la variété K 768-3. La variété témoin H 279-1 possède le rendement fibre à l'égrenage (RE) le plus faible.

Dans la zone 2 en effet, les variétés I 875-3 et K 768-3 ont donné les rendements en fibre les plus élevés. Elles sont suivies des variétés $\mathrm{H}$ 782-3 et K 787-2. Le plus faible rendement en fibre dans cette zone a été obtenu par la variété E 956-2.

Dans la zone 3, les variétés I 875-3 et K 768-3 ont montré les rendements en fibre les plus élevés comme en zone 2. La variété témoin $\mathrm{H}$ 279-1 s'est par contre révélée comme la moins performante en produisant le plus faible rendement fibre de la zone.

Dans la zone 4 enfin, la variété I 875-3 avec un rendement en fibre de $+2,33 \%$ par rapport au témoin H 279-1 s'est révélée comme la plus performante alors que la variété E 956-2 avec un rendement en fibre de-0,37\% par rapport à la variété témoin H 279-1 est apparue comme la plus faible des toutes les variétés comparées dans cette zone.

Tableau 6 : Rendement à l'égrenage de huit(8) variétés éprouvées de cotonnier dans les zones agro écologiques du Bénin.

\begin{tabular}{llllllllll}
\hline Site & \multicolumn{2}{c}{ Zone 1 } & \multicolumn{2}{c}{ Zone 2 } & \multicolumn{2}{c}{ Zone 3 } & \multicolumn{2}{c}{ Zone 4 } \\
\cline { 2 - 9 } & RE & Ecart & RE & écart & RE & écart & RE & écart \\
\hline H 279-1 & $43,38 \mathrm{f}$ & - & $43,69 \mathrm{c}$ & - & $41,32 \mathrm{~d}$ & - & $43,20 \mathrm{e}$ & -
\end{tabular}




\begin{tabular}{ccccccccc} 
E 956-2 & $43,95 \mathrm{e}$ & 0,57 & $43,00 \mathrm{~d}$ & $-0,69$ & $41,71 \mathrm{~d}$ & 0,39 & $42,83 \mathrm{e}$ & $-0,37$ \\
E 944-2 & $43,80 \mathrm{e}$ & 0,42 & $43,85 \mathrm{c}$ & 0,16 & $42,24 \mathrm{c}$ & 0,92 & $43,59 \mathrm{~d}$ & 0,39 \\
H 769-5 & $44,34 \mathrm{~d}$ & 0,96 & $44,03 \mathrm{c}$ & 0,34 & $42,52 \mathrm{~b}$ & 1,2 & $44,26 \mathrm{c}$ & 1,07 \\
H 782-3 & $44,59 \mathrm{c}$ & 1,21 & $44,67 \mathrm{~b}$ & 0,98 & $42,90 \mathrm{~b}$ & 1,58 & $44,31 \mathrm{c}$ & 1,11 \\
I 875-3 & $45,67 \mathrm{a}$ & 2,29 & $45,92 \mathrm{a}$ & 2,23 & $43,76 \mathrm{a}$ & 2,44 & $45,53 \mathrm{a}$ & 2,33 \\
K 768-3 & $45,13 \mathrm{~b}$ & 1,75 & $45,46 \mathrm{a}$ & 1,77 & $43,55 \mathrm{a}$ & 1,84 & $44,90 \mathrm{~b}$ & 1,70 \\
K 787-2 & $44,74 \mathrm{c}$ & 1,36 & $44,26 \mathrm{~b}$ & 0,57 & $42,69 \mathrm{~b}$ & 1,37 & $43,72 \mathrm{~d}$ & 0,52 \\
\hline Nbre rep & & & & & 4 & & & \\
Psite & & & & 0,95 & & & \\
Pvariété & 0,00 & & 0,00 & & 0,00 & 0,00 \\
F & $* *$ & & $* *$ & & $* *$ & $* *$ \\
\hline
\end{tabular}

CV : Coefficient de Variation et $* *$ : Hautement significatif au seuil de 0,01, Nbrerep: nombre de répétition, Les moyennes suivies des lettres alphabétiques différentes pour les mêmes caractéristiques sont significativement différentes $(p<0,05)$ selon le test de Scott-

Knott et celles suivies de la même lettre pour les mêmes caractéristiques ne sont pas significativement différentes $(\mathrm{p}<0,05)$ selon le test.

\section{Poids de 100 graines (Seed Index) des différentes variétés}

L'analyse de la variance des données relatives au poids de 100 graines a mis en évidence un une différence hautement significatif $(p=0,00)$ entre les sites. Ce qui signifie que les variétés se classent différemment d'un site à un autre (tableau 6).

En effet, dans les zones 1 et 3l'analyse de variance n'a révélé aucune différence entre les variétés étudiées. Par contre dans les zones 2 et 4 , les variétés sont significativement différentes. On peut noter que dans la zone 2,ce sont les variétés $\mathrm{K}$ 787-2, K 768-3 et $\mathrm{H}$ 782-3, qui ont les graines les plus grosses alors dans la zone 4, c'est la variété $\mathrm{K}$ 787-2 qui a produit les graines les plus grosses (tableau 7).

Tableau 7: Seed Index de huit (8) variétés éprouvées de cotonnier dans les zones agro écologiques.

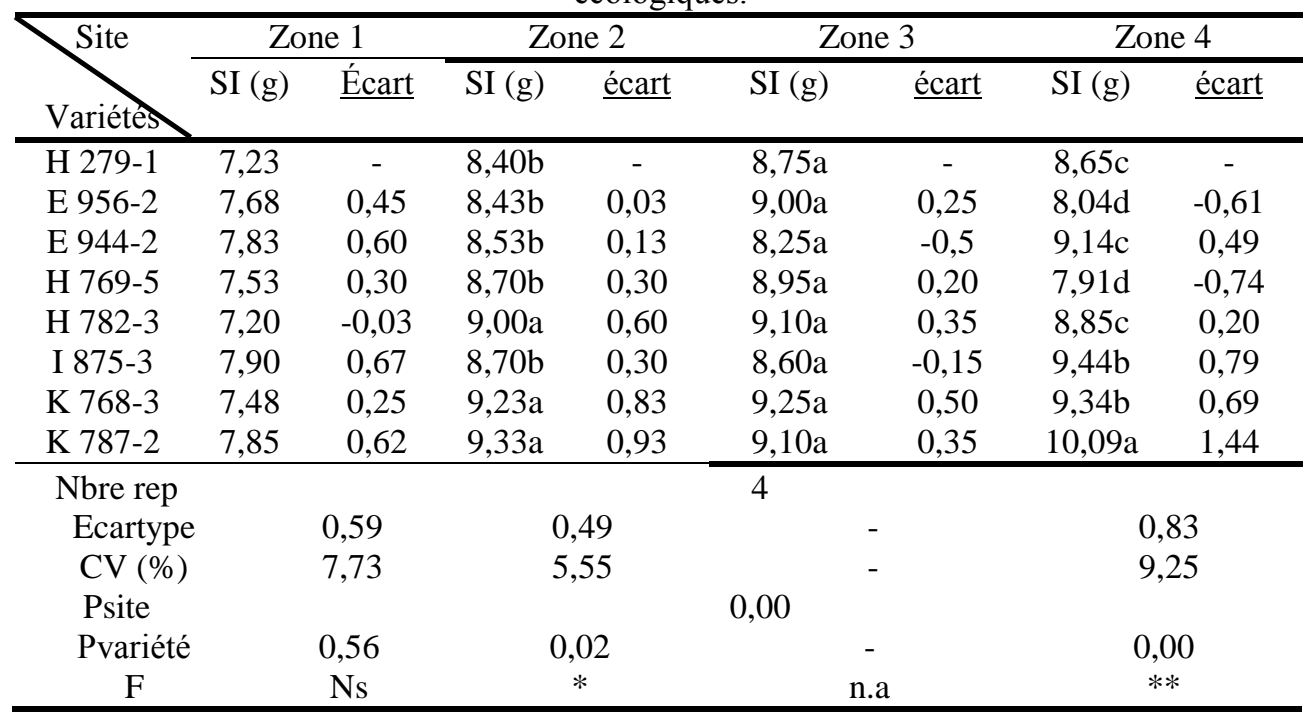


CV : Coefficient de Variation, ns : non significatif, * : Significatif au seuil de 0,05, Nbrerep: nombre de répétition, SI : seed index. Les moyennes suivies des lettres différentes pour les mêmes caractéristiques sont significativement différentes $(\mathrm{p}<0,05)$ selon le test de ScottKnott et celles suivies de la même lettre ne sont pas significativement différentes $(p<0,05)$.

\section{Identification des meilleures variétés dans chaque zone agro- écologique.}

\section{Bonnes variétés sur la base des résultats techniques}

De l'analyse des composantes agronomiques des huit variétés mises en comparaison dans les quatre aires de production cotonnière, on peut retenir que toutes les nouvelles variétés ont un rendement à l'égrenage supérieur à celui du témoin $\mathbf{H}$ 279-1 et de grosses graines. Pour ce qui est des rendements coton-graine au champ la plupart des nouvelles variétés sont supérieures au témoin et répondent aussi les exigences des égreneurs. Parmi toutes ces variétés, les plus productives par zone agro-écologique se présentent ainsi qu'il suit :

Dans la première zone (Alibori/Atacora), représentée par le site de Angaradébou, trois variétés ont un rendement coton graine supérieur à celui du témoin ; il s'agit de E 956-2 (+16,55\%), H 782-3 (+9,09\%) et $\mathbf{H ~ 7 6 9 - 5}$ $(+6,64 \%)$. Elles sont de ce fait les plus intéressantes pour les cotonculteurs.

Dans la deuxième zone (Borgou/Donga), représentée par le site de Okpara, trois variétés ont un rendement en coton graine supérieur à celui du témoin. Il s'agit des variétés $\mathbf{H} \mathbf{7 6 9 - 5}(+11,25 \%), \mathbf{K} \mathbf{7 6 8 - 3}(+4,54 \%)$ et $\mathbf{K}$ 787-2 (+2,82\%).

Dans la troisième zone (Collines), représentée par le site de Savalou, les variétés K 768-3 (+8,60 \%), E 944-2 (+8,08 \%) et H 769-5 (+7,37\%) sont significativement supérieures au témoin pour le rendement en coton graine.

Dans la quatrième zone (Zou/Plateau/Couffo), représentée par le site de Kétou, les variétés H 782-3 (+21,70\%), E 956-2 (+17,42 \%) et I 875-3 $(+8,18 \%)$, se sont avérées significativement supérieures au témoin.

En somme, on peut constater que la variété $\mathbf{H ~ 7 8 2 - 3}$ a été la plus homogène dans toutes les aires de production cotonnière du point de vue rendement au champ, mais l'objectif de cette étude n'est pas une variété moyenne mais des variétés spécifiques à chaque zone. Ainsi, les variétés les plus intéressantes du point de vue agronomique par zone agro écologique sont : E 956-2 pour la Zone1, $\mathbf{H}$ 769-5 pour la zone 2, $\mathbf{K}$ 768-3 pour la zone 3 et $\mathbf{H}$ 782-3 pour la zone 4. Cependant, l'étude économique permettra de confirmer ou d'infirmer que les variétés ainsi identifiées sur les aspects agronomiques, sont les meilleures pour chacune des zones préalablement définies. 


\section{Meilleures variétés par zone sur la base des résultats économiques}

A l'issue de l'étude économique (tableau 7), les variétés qui permettent de mieux rentabilisation la production cotonnière au Bénin se présentent comme suit :

Au niveau national, toutes zones confondues, $\mathbf{H}$ 782-3 est la meilleure variété moyenne. Elle apporte une plus-value importante à la filière $(13,72$ milliards FCFA) dont 3,99 milliards FCFA aux Cotonculteurs et 9,73 milliards FCFA aux autres acteurs y compris les égreneurs. Mais, elle n'est forcément pas la meilleure dans chacune des zones agro écologiques.

Dans la zone 1 ; la variété E 956-2 est la meilleure car en plus de ses qualités agronomiques, elle apporte une plus-value importante (16,38 milliards de FCFA) à la filière dont 6,10 milliards de F CFA aux Cotonculteurs et 10,28 milliards de FCFA aux autres acteurs y compris les égreneurs (tableau 8).

Dans la zone 2 ; la variété H 769-5 est la meilleure car elle apporte une plus-value importante (5,42 milliards de FCFA) à la filière dont 2,19 milliards de FCFA aux Cotonculteurs et 3,23 milliards de FCFA aux autres acteurs y compris les égreneurs (tableau 8).

Dans la zone 3, la variété K 768-3 est la meilleure car elle apporte une plus-value importante $(3,10$ milliards de F CFA) à la filière dont 0,96 milliard de FCFA aux Cotonculteurs et 2,14 milliards de FCFA aux autres acteurs.

Dans la zone 4 ; la variété $\mathbf{H ~ 7 8 2 - 3}$ est la meilleure car elle apporte une plus-value importante (2,00 milliards de FCFA) à la filière dont 0,82 milliard de FCFA aux Cotonculteurs 1,18 milliards de FCFA aux autres acteurs.

Tableau 8: Incidence économique des nouvelles variétés sur les zones et sur le pays

\begin{tabular}{|c|c|c|c|c|c|c|}
\hline \multirow{2}{*}{$\begin{array}{l}\text { Variétés par zones } \\
\text { Éléments de calcul }\end{array}$} & \multicolumn{2}{|c|}{ Pays } & \multirow{2}{*}{$\begin{array}{c}\text { Z1 } \\
\text { E 956-2 } \\
\end{array}$} & \multirow{2}{*}{$\begin{array}{c}\mathrm{Z} 2 \\
\mathrm{H} 769-5\end{array}$} & \multirow{2}{*}{$\frac{\mathrm{Z3}}{\mathrm{K} 768-3}$} & \multirow{2}{*}{$\begin{array}{c}\text { Z4 } \\
\text { H 782-3 }\end{array}$} \\
\hline & H 279-1 & $\begin{array}{c}\mathrm{H} \\
782-3\end{array}$ & & & & \\
\hline \multicolumn{7}{|l|}{ Données techniques } \\
\hline $\begin{array}{l}\text { Rendement (Kg/ha) } \\
\text { Rendement égrenage }(\%)\end{array}$ & $\begin{array}{r}1543 \\
42,84\end{array}$ & $\begin{array}{l}1625 \\
44,08\end{array}$ & $\begin{array}{l}1984 \\
43,95\end{array}$ & $\begin{array}{l}1820 \\
44,03\end{array}$ & $\begin{array}{r}1735 \\
43,55\end{array}$ & $\begin{array}{l}1534 \\
44,31\end{array}$ \\
\hline $\begin{array}{c}\text { Rendement graine }(\%) \\
\text { Perte en usine }(\%)\end{array}$ & $\begin{array}{c}54,16 \\
3,0\end{array}$ & $\begin{array}{c}52,92 \\
3,0\end{array}$ & $\begin{array}{c}53,05 \\
3,0\end{array}$ & $\begin{array}{c}52,97 \\
3,0\end{array}$ & $\begin{array}{c}53,45 \\
3,0\end{array}$ & $\begin{array}{c}52,69 \\
3,0\end{array}$ \\
\hline \multicolumn{7}{|c|}{ Hypothèses de prix } \\
\hline \multicolumn{2}{|c|}{ Part de marché en coton graine (\%) } & 100 & 53 & \multirow[b]{2}{*}{78000} & 16 & \multirow[b]{2}{*}{15000} \\
\hline Production & & 300000 & 159000 & & 48000 & \\
\hline $\begin{array}{l}\text { Coton graine } \\
\text { (FCFA/tonne) }\end{array}$ & 250000 & $\begin{array}{l}250 \\
000\end{array}$ & 250000 & 250000 & 250000 & 250000 \\
\hline & 1130 & 1130 & 1130 & 1130 & 1130 & 1130 \\
\hline Fibre (FCFA/tonne) & 000 & 000 & 000 & 000 & 000 & 000 \\
\hline
\end{tabular}


Marge financière brute par secteur (Milliard F CFA)

\begin{tabular}{|c|c|c|c|c|c|c|}
\hline \multicolumn{7}{|c|}{170,0} \\
\hline Filière (fibre + graine) & 156,28 & 0 & 100,12 & 46,76 & 27,54 & 9,87 \\
\hline Coton graine & 75,00 & 78,99 & 45,85 & 21,69 & 13,03 & 4,57 \\
\hline Egrenage et autres activités & 81,28 & 91,01 & 54,27 & 25,06 & 14,51 & 5,31 \\
\hline \multicolumn{7}{|c|}{ Gain financier brut par catégorie d'acteurs(Milliard F CFA) } \\
\hline Filière & & \multirow{3}{*}{$\begin{array}{c}13,72 \\
3,99 \\
9,73\end{array}$} & 16,38 & 5,42 & 3,10 & 2,00 \\
\hline Cotonculteurs & & & 6,10 & 2,19 & 0,96 & 0,82 \\
\hline Egreneurs, transporteurs e & autres. & & 10,28 & 3,23 & 2,14 & 1,18 \\
\hline
\end{tabular}

La meilleure variété par zone agro-écologique est identifiée (Figure 1), grâce à l'étude économique inspirée de l'évaluation de l'incidence économique sur la filière coton du remplacement de la variété STAM 18 A par la variété H 279-1 (Hougni et al., 1999). 


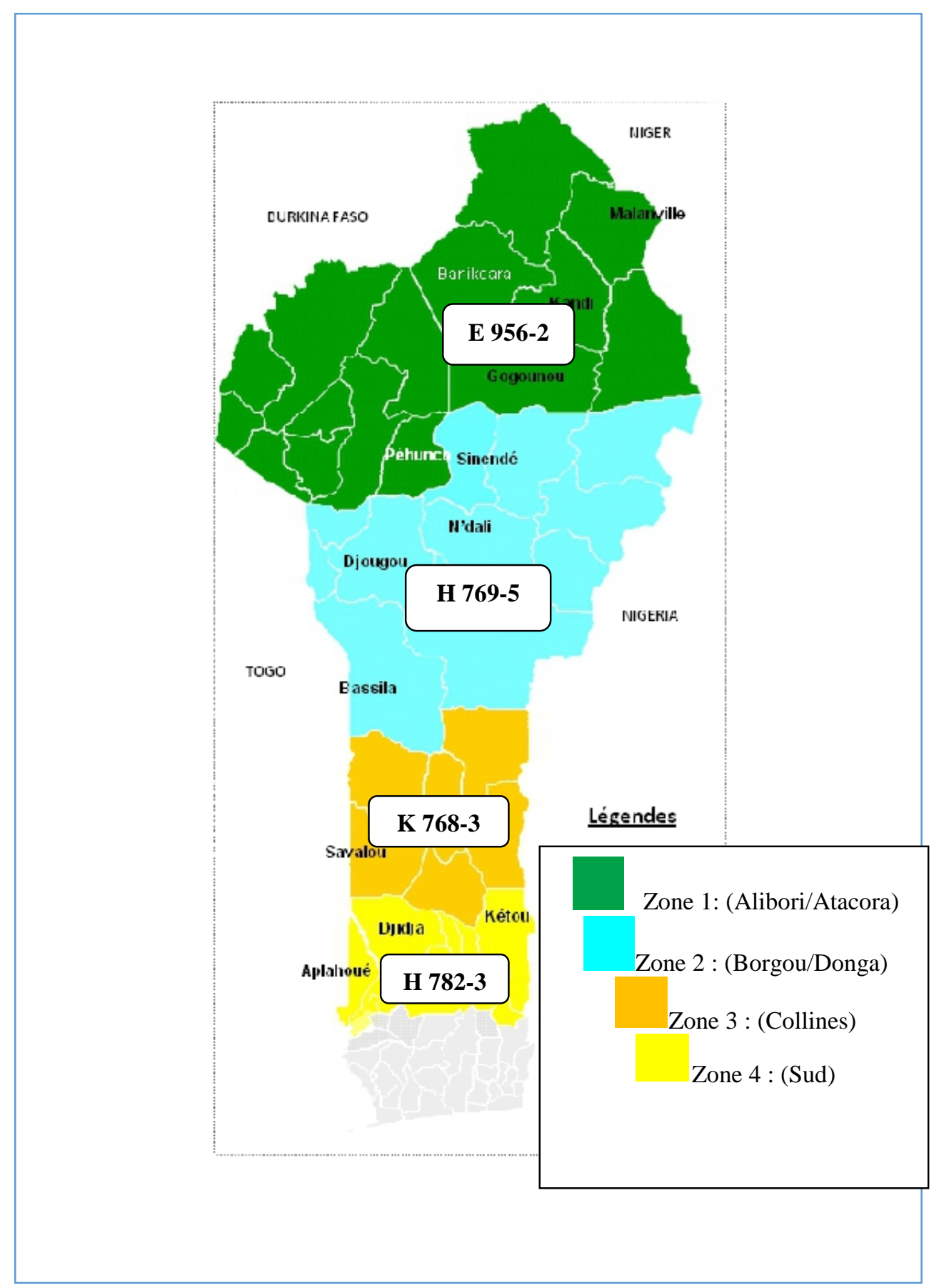

Figure 1: Carte montrant la variété adaptée à chaque zone agroécologique du Bénin Source : CRA-CF

\section{Discussion}

Les résultats obtenus au cours de cette étude ont donné des différences significatives sur beaucoup de paramètres d'un site à un autre. Cela explique 
une fois encore la pertinence de la régionalisation variétale. Ainsi, ce travail qui a porté sur la caractérisation agro morphologique des variétés éprouvées et l'identification des variétés adaptées à chaque zone agro-écologique est un point de départ non moins important.

En effet, les différences significatives observées d'un site à un autre pourraient s'expliquer en partie par les conditions pluviométriques qui varient d'une zone agro-écologique à une autre. Nos travaux ont montré que les rendements sont différents d'une zone agro écologique à une autre et décroissent du Nord au Sud. Ainsi, les plus forts rendements ont été obtenus à Angaradébou dans la zone let les plus faibles à Kétou dans la zone 4 alors que les rendements intermédiaires ont été obtenus à Okpara (zone 2) et à Savalou (zone 3). Nos résultats viennent appuyer le gradient Nord-Sud révélé par les études antérieures sur le zonage (Hougni et al., 2001). Nos résultats sont également en harmonie avec ceux trouvés lors des dernières campagnes (Rapport de campagnes, 2010).

Les rendements au champ obtenus à l'issue de cette étude nous ont permis de conclure que la variété $\mathrm{H}$ 782-3 a été la plus stable dans toutes les zones agro-écologiques. De ce fait, elle devient la plus intéressante qui pourrait remplacer la variété en vulgarisation à cause de la masse financière qu'elle engendre, estimée à près de 13 milliards supplémentaire par rapport au témoin en vulgarisation si la politique de variété unique devait être poursuivie. Notre étude a permis de montrer que la plupart des nouvelles variétés sont supérieures au témoin. Ces résultats sont en harmonie avec ceux trouvés par Sèkloka et al., (2009) dans leurs études sur deux variétés prometteuses pour la filière coton au Bénin. Ces auteurs ont montré que toutes les lignées éprouvées sont meilleures au témoin. Cependant, ces derniers n'ont travaillé que sur trois variétés dont le témoin (H 279-1) contrairement à nos travaux qui ont porté sur huit (8) variétés éprouvées créées au Bénin. Toutefois, il faut noter que nos résultats sont quelque peu différents de ce qu'ils ont trouvé. Pour preuve, la variété I 875-3 contre toute attente ne s'est pas révélée meilleure en termes de rendement au champ mais plutôt en termes de rendement à l'égrenage où elle a gagné près de $5 \%$ de plus que le témoin dans presque toutes les zones.

Nos résultats ont aussi montré que le pourcentage de fibre et le poids de 100 graines (SI) sont faiblement liés et évoluent en sens inverse. Ces résultats corroborent bien ceux trouvés par Lançon et al.,(1995). Leurs travaux ont montré que ces paramètres évoluent en sens inverse sous l'effet de la sélection comme sous l'effet du milieu. En effet, le rendement au champ est beaucoup plus corrélé avec la hauteur de la plante comme l'ont montré les travaux de Lançon etal, (1995). L'évaluation de la grosseur des graines (Seed Index) dans les zones let 2 a permis de constater que toutes les variétés ont des graines à peu près du même poids. Ces résultats sont en harmonie avec 
ceux trouvés par Sèkloka et al.,(2009) dans leurs études sur deux variétés prometteuses de coton.

Suivant les hypothèses de prix retenues dans l'analyse économique le remplacement de la variété H 279-1 par la variété H 782-3 pourrait générer une plus-value de 13,72 milliards pour la filière et bien réparties entre les cotonculteurs, les égreneurs et les autres acteurs. Ces valeurs sont supérieures à celles trouvées par Sèkloka et al., (2009) du fait de la revalorisation du prix des fibres de coton sur le marché international et de la régionalisation des variétés. Notre étude a porté sur huit (8) variétés. Cette gamme de variétés nous a permis d'identifier une variété spécifique à chaque zone agro écologique. Ainsi, du Nord au Sud en passant par le Centre, les variétés spécifiques du fait de leur rendement au champ, de leur rendement fibre à l'égrenage et du coût de la fibre sur le marché international en 2012, sont $\mathrm{E}$ 956-2, H 769-5, K 768-3 et $\mathrm{H}$ 782-3 respectivement pour les zones 1, 2, 3 et 4. Cette idée de régionalisation apporterait une plus-value de 26,9 milliards à la filière avec une hypothèse réaliste de production de 300000 tonnes de coton-graine. Tout ceci nous amène à dire que la régionalisation est donc opportune et apportera une plus-value substantielle à chacun des acteurs de la filière. Ainsi, la politique de variété unique doit laisser place à la régionalisation.

\section{Conclusion et recommandations}

A l'issue de cette étude, il ressort que le Bénin regorge de potentialités en termes de diversification variétale pour la production de coton (or blanc). En effet, les variétés mises en comparaison dans les différentes zones agro écologiques productrices de coton se sont effectivement comportées de différentes manières et se sont montrées pour la plupart supérieures au témoin en donnant chacune des résultats satisfaisants quant au rendement en coton graine, au rendement en fibre, au rendement à l'égrenage avec de bonne précocité de production. Aussi, l'analyse de l'incidence économique de la spécificité variétale de la production cotonnière a montré l'importance de la régionalisation par la plus-value qu'apporterait cette nouvelle politique variétale si elle était mise en œuvre.

\section{References:}

1. Ashokkumar, K., Ravikesavan, R. (2011). Morphological diversity and per se performance in upland cotton (Gossypium hirsutum L.). J. Agric. Sci. 3(2), pp: 107-113.

2. CRA-CF, (2005). Rapport de campagne, Agronomie, CRA-CF, MAEP Bénin, $84 \mathrm{p}$. 
3. CRA-CF, (2010). Rapport de campagne, Agronomie CRA -CF, MAEP Bénin, $103 \mathrm{p}$.

4. Demol, J. (1992). Connaissance de la plante. In : Cotonnier au Zaïre. Publication du service agricole $\mathrm{N}^{\circ} 29$, Administration générale de la coopération au développement, Bruxelles, pp. $17-26$.

5. Esteulle, B. et Perenne, J. (1996). Production cotonnière africaine campagne 2005-2006. Note de conjoncture, p. 2.

6. Hazard, (2003). Le coton en Afrique de l'Ouest et du centre et son insertion dans les échanges internationaux. ENDA Prospectives, Dialogues, Politiques, Dakar

7. Kpadé, C. P. (2011). Adaptation de la coordination et nouvelles contradictions entre acteurs du système coton au Bénin face à la libéralisation économique. Economies et finances. Thèse de doctorat, Université de Bourgogne, France), 372 p.

8. Hougni, A. (2009). Qualités et valorisation du coton fibre d'Afrique Zone Franc (AZF) dans les échanges internationaux. Doctorat/Thèse Unique/PhD, Université de Bourgogne, Dijon, France, Nov. 2009, 320 $\mathrm{p}$

9. Hougni, A., (2004). Contribution à l'analyse des problèmes de qualité dans la filière coton au Bénin; le sous-système semence : les acteurs et leurs rôles. Mémoire de DEA, Université de Bourgogne, Dijon, France, $96 \mathrm{p}$.

10. Hougni, A., Sêkloka, E., Djaboutou, M., Lançon, J. (2001). Au Bénin, la qualité du coton graine varie suivant les quatre zones agroécologiques : Seed index, rendement à l'égrenage, longueur, micronaire et couleur, In: Actes des journées coton du Cirad : Montpellier, 23 et 24 juillet 2001. Montpellier : CIRAD-CA, pp : 8186.

11. Hougni, A., Djaboutou, M., Sêkloka, E., Lançon, J. (1999). Stam F, Stam 18 A et H 279-1 : variétés passées, présentes ou à venir pour la filière coton du Bénin? Synthèse des résultats obtenus entre 1991 et 1998, Actes des journées coton du Cirad. Montpellier, du 19 au 23 Juillet 1999. Montpellier : CIRAD-CA, pp : 124-139.

12. Lancon, J., (1995). Effet de la densité de semis en sélection sur l'amélioration génétique du cotonnier: interaction, structures de corrélations, hétérosis et valeur en lignées. Thèse de Doctorat, Paris, Université de Paris-Orsay, 119 p.

13. Matthess, A., Van den Akker, E., Chougourou, D. \& Midingoyi Jun, S. (2005). Le coton au Bénin : Compétitivité et durabilité de cinq systèmes culturaux cotonniers dans le cadre de la filière, BMZGTZ/MAEP, Cotonou. $201 \mathrm{p}$ 
14. Sèkloka, E., Hougni, A., Katary, A., Djaboutou, C. M. et Lançon, J. (2009). I 875.3 et H 769.5, variétés prometteuses de coton (Gossypium hirsutumL.) sélectionnées au Bénin. Bulletin de la Recherche Agronomique du Bénin, nº3, pp : 48-57.

15. Sêkloka, E., Lançon, J., Djaboutou, M., Lewicki-Dhainaut, S., Takpara, D., Assogba, L., Orou Mousse, B. I., (2001). La sélection participative : impliquer les utilisateurs dans l'amélioration des plantes. Actes de l'atelier - Montpellier, 5-6 septembre 2001; Montpellier, France, CIRAD-MICAP, pp : 56-63. 